\title{
Avaliação da qualidade de conservação de vacinas na Atenção Primária à Saúde
}

\author{
Assessment of quality of vaccine storage and conservation \\ in primary health care centers
}

Valéria Conceição de Oliveira ${ }^{1}$

Maria Del Pilar Serrano Gallardo ${ }^{2}$

Ricardo Alexandre Arcêncio ${ }^{3}$

Tarcísio Laerte Gontijo ${ }^{1}$

Ione Carvalho Pinto ${ }^{3}$

${ }^{1}$ Departamento de Enfermagem, Universidade Federal de São João Del-Rei. Av. Sebastião Gonçalves Coelho, Chanadour. 35.501296 Divinópolis MG Brasil. valeriaoliveira@ufsj.edu.br

${ }^{2}$ Universidade Autônoma de Madrid.

${ }^{3}$ Escola de Enfermagem de Ribeirão Preto, Universidade de São Paulo.
Abstract This is an evaluative study of the quality of vaccine storage and conservation in primary health care centers (PHC) in the mid-west region of the state of Minas Gerais. Dimensions, structures and processes were the criteria used to measure the level of quality in 261 vaccine storage and conservation units in over 55 municipalities in the area. Quality levels were defined by means of a scoring system with different weighted scores attributed to indicators for each dimension being rated. Categories for quality levels were then defined as: "adequate," "inadequate" and "critical." Pearson's chi-square test was used to verify the correlation between quality level and population size and adherence to the Unified Health System. It was observed that vaccine storage and conservation quality levels were inadequate in 59.3\% and critical in 26.9\% of these facilities. Small municipalities that are registered for Full Primary Health Care Management featured the worst vaccine storage facilities. Vaccine conservation supervision, which spans a series of activities present in nursing praxis, indicates the need to train human resources, monitor and assess work processes and conduct further studies in the field.

Key words Cold storage, Vaccines, Health service evaluation, Quality of health care, Nursing
Resumo Estudo avaliativo do grau de qualidade da conservação de vacina nas Unidades de Atenção Primária à Saúde da região Centro-Oeste do Estado de Minas Gerais. Foram utilizados critérios das dimensões, estrutura e processo para mensurar o grau de qualidade das 261 salas de vacina distribuídas nos 55 municípios da região. O grau de qualidade foi definido por meio de um sistema de escores com pesos diferenciados para indicadores em cada dimensão avaliada sendo definidas as categorias: "adequado", "não adequado" e "crítico". Para verificar a relação entre o grau de qualidade e o porte populacional e adesão ao SUS utilizou-se o teste do qui-quadrado de Pearson. Observou-se que o grau de qualidade de conservação de vacina não era adequado em 59,3\% e 26,9\% apresentaram grau crítico de qualidade. Os municípios de pequeno porte habilitados na Gestão Plena da Atenção Básica concentraram as piores salas de vacina. O cuidado com a conservação de vacinas aponta para a necessidade de capacitação dos recursos humanos, de monitoramento e avaliação do processo de trabalho e de novos estudos na área. Palavras-chave Rede de frio, Vacinas, Avaliação de serviços de saúde, Qualidade da assistência à Saúde, Enfermagem 


\section{Introdução}

A vacinação constitui ação prioritária de Atenção Primária à Saúde (APS) e de grande impacto nas condições gerais de saúde da população. Representa um dos avanços da tecnologia médica nas últimas décadas constituindo-se no procedimento de melhor relação custo e efetividade no setor saúde ${ }^{1}$.

O Programa Nacional de Imunização (PNI) do Brasil é considerado um dos mais completos dentre os países em desenvolvimento, tendo sido pioneiro na introdução de algumas vacinas e demonstrado alta capacidade técnica nas questões de logística para imunização². Contudo, apesar dos bons resultados do PNI, estudos ${ }^{3-6}$ apontam deficiências em salas de vacina, no Brasil, principalmente relacionadas à conservação dos imunobiológicos. Essas deficiências podem provocar aumento considerável nos custos do PNI devido a perdas desnecessárias de vacinas por erros de manutenção da cadeia de frio, além de comprometer a efetividade do programa.

Deve-se considerar que prejuízos na qualidade da vacina por procedimento inadequado no armazenamento, transporte ou manipulação são considerados perda evitável que, geralmente, estão relacionados à falta de manutenção preventiva/corretiva dos equipamentos e desconhecimento e descumprimento dos profissionais que trabalham em sala de vacina sobre as normas de conservação dos imunobiológicos?.

Também a inativação dos imunobiológicos não é identificada por alterações nas características físicas, o que pode comprometer a proteção das doenças a qual se destina. A ausência do seu efeito, ou seja, a não proteção contra a doença, poderá ser rotulada como falha primária ou secundária, e o papel da inadequada conservação da vacina não será identificado ${ }^{8}$.

Assim, uma recente investigação nos Estados vem chamando atenção para a importância da conservação de vacina para o controle das doenças imunopreveníveis, pois, levanta a hipótese de que falhas no armazenamento de vacinas em unidades de saúde locais podem estar contribuindo para um recente aumento nas taxas de morbidade da coqueluche no país?.

A preocupação com a qualidade da imunização sempre fez parte das propostas da política da APS, assinalando para aspectos de prevenção de doenças e/ou proteção específica. Com a descentralização dos serviços de saúde a partir da Lei 8.080/1990, a ampliação das responsabilidades municipais no tocante à saúde facilitou a rea- lização e a coordenação das atividades do PNI no nível local ${ }^{4}$. Portanto, a descentralização da atenção à saúde transferiu a gestão das unidades responsáveis pela vacinação para os municípios. Com isso, a manutenção de pessoal capacitado e o suprimento de materiais necessários para o armazenamento e o transporte dos insumos ficaram a cargo dos mesmos ${ }^{8}$.

Nesta perspectiva, o processo de avaliação torna-se imprescindível para monitoramento dos serviços de saúde e constitui instrumento essencial de apoio, pela sua capacidade de fornecer elementos de conhecimento que subsidiem a tomada de decisão, propiciando o aumento da eficiência, eficácia e efetividade das atividades desenvolvidas pelo serviço ${ }^{10}$. Assim, este artigo tem como objetivo avaliar a qualidade da conservação de vacina nas Unidades de Atenção Primária à Saúde da macrorregião Oeste de saúde do Estado de Minas Gerais.

\section{Método}

Trata-se de pesquisa avaliativa de análise de qualidade, realizada na macrorregião Oeste de saúde do estado de Minas Gerais entre 2010 e 2011. Essa macrorregião é uma das treze de Minas Gerais e está dividida em seis microrregiões. É composta por 55 municípios que juntos possuem população estimada pelo Instituto Brasileiro de Geografia e Estatística (IBGE) ${ }^{11}$ de 1,2 milhões habitantes em 2012, correspondendo a 6,1\% da população mineira.

Para selecionar as dimensões e os critérios a serem utilizados neste estudo elaborou-se um modelo lógico ${ }^{12}$, correspondente a uma imagem -objetivo, visando funcionar como referência para a apreciação do grau de qualidade da conservação de vacinas nas UAPS. A imagem-objetivo contemplou os atributos de estrutura e processo necessários para a efetiva conservação de vacinas.

Para cada um dos atributos descritos no modelo lógico, foram definidos os insumos necessários, as atividades, os produtos e os resultados esperados, levando-se em consideração a interação dos efeitos de seus componentes e o seu impacto sobre a conservação de vacina.

Na dimensão estrutura, utilizou-se como critérios de qualidade os itens: existência de refrigerador exclusivo; existência de termômetro de máxima e mínima; presença de gelo reciclável no congelador; presença de garrafas com água na parte inferior do congelador; ausência de objetos 
na porta do refrigerador; e, profissional capacitado em sala de vacina e/ou rede de frio.

Já na dimensão processo definiu-se três componentes: a) organização do refrigerador; b) monitoramento da temperatura e; c) condutas frente às alterações de temperatura. A seguir estão listados os critérios de qualidade utilizados em cada um desses componentes:

Organização do refrigerador: distribuição correta dos imunobiológicos segundo prateleiras; realização de limpeza e degelo corretos; existência de manutenção preventiva e/ou corretiva para o refrigerador;

Monitoramento da temperatura: realização de leitura e registros corretos das temperaturas no início e no final da jornada de trabalho; realização de ambientação de bobinas de gelo reciclável; e, realização de monitoramento da temperatura da caixa térmica ou do equipamento de uso diário;

Condutas frente à alteração de temperatura: comunica imediatamente a instância superior em casos de alteração; preenche e envia o formulário de avaliação de imunobiológicos sob suspeita; manutenção de vacinas sob suspeitas em temperaturas adequadas até pronunciamento de instância superior.

O modelo lógico construído subsidiou a definição das perguntas avaliativas e a partir delas os critérios utilizados na análise da estrutura e do processo. A avaliação de resultados não integra este estudo. Definiu-se para cada critério das dimensões estrutura e processo o método de cálculo, os parâmetros e as técnicas de coleta, constituindo assim, a matriz de medidas. Estes critérios foram definidos através de consultas a especialistas, uma vez que não há normas que os defina. Na apreciação normativa a opinião de especialista é considerada uma norma, podendo os especialistas fornecer normas de qualidade ${ }^{12}$.

Baseado na literatura sobre o assunto e no consenso dos especialistas elaborou-se uma matriz de medidas para avaliar o grau de qualidade da conservação de vacina nas 261 salas de vacina das UAPS da região. Atribuiu-se 10 pontos para a dimensão estrutura e 25 para a do processo, totalizando 35. Esses pontos foram distribuídos em cada dimensão e componentes, constituindo-se na matriz de análise e julgamento para a avaliação do grau de qualidade da conservação de vacina.

$\mathrm{Na}$ dimensão estrutura, os critérios mais valorizados com pontuação máxima de 3,0 pontos foram aqueles considerados essenciais para a conservação de vacina nas UAPS: profissional capacitado e presença de termômetro no refrigerador da sala de vacina.
No processo, os critérios avaliados com pontuação máxima de 5,0 pontos foram: o armazenamento na primeira prateleira com somente as vacinas que podem ser submetidas à temperatura negativa; comunica imediatamente a instância hierarquicamente superior em casos de alterações de temperatura; e, realização de leitura e registro corretos das temperaturas no início e no final da jornada de trabalho. Utilizou-se ainda dados sobre o porte populacional e a condição de gestão do município em análise.

Como instrumento de coleta dos dados utilizou-se o questionário do Programa de Avaliação do Instrumento de Supervisão para Sala de Vacina - PAISSV 2.0 do Programa Nacional de Imunização/MS ${ }^{13}$. O questionário foi aplicado no período de 2010 a $2011 \mathrm{em}$ todas as 261 salas da região, sendo respondido por um enfermeiro ou técnico/auxiliar de enfermagem atuante na Sala de Vacina. O questionário foi aplicado pela responsável técnica da imunização da Superintendência Regional de Saúde (SRS), responsável pela macrorregião oeste de saúde. A mesma compareceu em todas as 261 salas da região para aplicação do questionário. Foram excluídas oito salas por não contar com a presença do enfermeiro ou auxiliar/técnico de enfermagem no momento da visita para a coleta de dados.

A variável porte populacional foi obtida nas bases de dados do Instituto Brasileiro de Geografia e Estatística (IBGE) e a condição de habilitação dos municípios ao SUS foi obtido nas bases de dados da Secretaria Estadual de Saúde de Minas Gerais.

Os dados coletados foram inseridos no programa Epi-data 6.0 e analisados no Statistical $\mathrm{Pa}$ ckage for the Social Sciences (SPSS) 13.0. As informações foram registradas em dupla entrada, para eliminar possíveis erros de digitação.

Depois de atribuída a pontuação correspondente aos itens avaliados em cada dimensão, estes foram totalizados e assim ficaram constituídos os escores de cada uma das dimensões (estrutura e processo).

Dessa forma, procedeu-se à construção dos escores do grau de qualidade considerando três etapas. Na primeira, determinou-se o grau de qualidade de cada dimensão ( $\sum$ dos pontos em cada dimensão). Em seguida, calculou-se o grau de qualidade de cada um dos componentes da dimensão processo ( $\sum$ dos pontos em cada componente). Por último, os valores observados em cada uma das dimensões foram somados gerando o grau de qualidade total ( $\sum$ observados $/ \Sigma$ dos pontos máximos x 100) da conservação de 
vacinas nas 253 salas da região. A classificação do grau de qualidade total de conservação de vacina estabelecidos foram: (1) crítico: $<60,0 \%$; (2) não adequado: $\geq 60,0$ e $<90,0$; (3) adequado: $\geq 90,0$.

Realizou-se ainda a análise de associação da qualidade da conservação dos imunobiológicos nos diferentes municípios estudados, utilizando-se variáveis como o porte populacional e a condição de gestão do Sistema Único de Saúde (SUS). Classificou-se o porte populacional dos municípios em pequeno (menos de $10 \mathrm{mil} \mathrm{habi-}$ tantes), médio (de 10 a 50 mil habitantes) e grande (maiores de 50 mil habitantes). Já em relação à condição de gestão, os municípios foram divididos em habilitados na Gestão Plena do Sistema Municipal (GPSM) e na Gestão Plena da Atenção Básica Ampliada segundo a Norma Operacional da Assistência a Saúde(NOAS-SUS/2002).

Para a análise dos dados, procedeu-se a análise univariada com cálculo das frequências, medidas de posição (media, mediana) e dispersão. No trabalho, considerou-se o teste qui-quadrado de Pearson para proporções, sendo as variáveis analisadas duas a duas, primeiro, a qualidade de conservação e o porte do município, e na sequência a qualidade de conservação com condição de gestão. Cabe destacar que os municípios foram categorizados em grupo, levando em consideração o porte populacional e a condição de gestão. A suposição de independência entre os grupos e observações em frequência ou contagem estiveram satisfeitas. Os autores trabalharam com as seguintes hipóteses: $\mathrm{H}_{0}$ as frequências observadas não são diferentes das esperadas, portanto não existem diferenças entre as frequências dos grupos. $\mathrm{H}_{1}$ As frequências observadas são diferentes da frequências esperadas, portanto existe diferença entre as frequências dos grupos. Admitiuse a probabilidade do erro tipo I, de $5 \%$.

O estudo foi aprovado pelo Comitê de Ética em Pesquisa do Hospital São João de Deus.

\section{Resultados}

Analisou-se todas as salas de vacinas das microrregiões de Bom Despacho, Itaúna e Formiga e nas microrregiões de Pará de Minas, Divinópolis e Campo Belo as proporções de salas avaliadas foi de $97,9 \%, 94,9 \%$ e $94,1 \%$ respectivamente.

Das 253 salas de vacina avaliadas $62(24,5 \%)$ são exclusivas para imunização. A maioria das salas, 213 (84,2\%), localizam-se em Unidades de Saúde da Família e 40 (15,8\%) em Unidade Básicas de Saúde tradicionais. Quase metade das sa- las de vacina, $127(49,8 \%)$ atendem a população da zona urbana, $30(11,9 \%)$ atendem população de zona rural e $96(38,3 \%)$ acolhem população mista.

Quanto ao funcionamento das salas de vacina da região, $209(83,0 \%)$ funcionam oito horas diárias e o restante de 4 a 6 horas. Duas salas de vacina avaliadas possuíam geladeira especial (câmara) e o restante contava com refrigerador tipo doméstico.

Diante dos critérios adotados, esta avaliação demonstrou que somente 35 (13,8\%) salas de vacina alcançaram grau de qualidade total adequado e que $150(59,3 \%)$ foram classificadas como não adequadas. As demais 68 (26,9\%) se enquadraram como críticas.

Registrou-se que as microrregiões de Divinópolis e Bom Despacho concentram um número maior de salas de vacina com grau de qualidade adequado. Já as microrregiões de Pará de Minas e Formiga agrupam as salas de vacinas mais críticas (Tabela 1).

Das 253 salas avaliadas, 106 (41,9\%) localizam-se em municípios com mais de 50 mil habitantes, 100 (39,5\%) em municípios com população estimada entre 10 e 50 mil habitantes e o restante, 47 (18,6\%), em cidades com menos de 10 mil habitantes. Analisando o grau de qualidade das salas de vacinas em relação ao porte populacional do município onde esta inserida, identifica-se que a maioria das salas classificadas como críticas $(48,9 \%)$ situam-se em municípios de pequeno porte. Já em relação à condição de gestão, $187(73,9)$ dos municípios são habilitados como GPABA e 66 (26,1\%) como GPSM. Identifica-se também maior proporção de salas críticas nos municípios habilitados como GPABA. Segundo análise univariada, identifica-se associação com significância estatística entre as variáveis, porte populacional e condição de gestão do município em relação ao grau de qualidade da conservação de vacina. Sendo assim, ser municípios de pequeno porte e habilitado em Gestão Plena da Atenção Básica Ampliada está associado à presença de maior número de salas de vacinas com grau de qualidade crítico (Tabela 2).

A Tabela 3 apresenta o grau de qualidade de cada uma das dimensões e também dos componentes da dimensão processo separadamente. Isto torna possível destacar os principais entraves na conservação dos imunobiológicos. Na estrutura, $90(35,6 \%)$ das salas apresentaram grau de qualidade adequado, e no processo $74(29,2 \%)$. É notável uma concentração de salas analisadas no grau de qualidade não adequado em estrutura $\mathrm{e}$ 
Tabela 1. Distribuição do grau de qualidade da conservação de vacina em municípios da macrorregião Oeste segundo as microrregiões, Minas Gerais, 2011.

\begin{tabular}{|c|c|c|c|c|c|c|}
\hline \multirow{3}{*}{ Microrregião de Saúde } & \multicolumn{6}{|c|}{ Grau de qualidade total } \\
\hline & \multicolumn{2}{|c|}{ Adequado } & \multicolumn{2}{|c|}{ Não adequado } & \multicolumn{2}{|c|}{ Crítico } \\
\hline & $\mathrm{N}$ & $\%$ & $\mathrm{~N}$ & $\%$ & $\mathbf{N}$ & $\%$ \\
\hline Divinópolis & 16 & 21,3 & 38 & 50,7 & 21 & 28,0 \\
\hline Bom Despacho & 4 & 17,4 & 13 & 56,5 & 6 & 26,1 \\
\hline Campo Belo & 6 & 12,5 & 35 & 72,9 & 7 & 14,6 \\
\hline Formiga & 4 & 12,1 & 18 & 54,6 & 11 & 33,3 \\
\hline Itaúna & 4 & 10,7 & 21 & 75,0 & 3 & 14,3 \\
\hline Pará de Minas & 1 & 2,2 & 25 & 54,3 & 20 & 43,5 \\
\hline
\end{tabular}

Tabela 2. Grau de qualidade da conservação de vacina segundo o porte populacional e a condição de gestão de municípios da macrorregião Oeste do estado de Minas Gerais, 2011.

\begin{tabular}{|c|c|c|c|c|c|c|c|}
\hline \multirow{3}{*}{ Critérios avaliados } & \multicolumn{6}{|c|}{ Grau de qualidade } & \multirow{3}{*}{$\mathrm{p}$ (valor) } \\
\hline & \multicolumn{2}{|c|}{ Adequado } & \multicolumn{2}{|c|}{ Não adequado } & \multicolumn{2}{|c|}{ Crítico } & \\
\hline & $\mathbf{N}$ & $\%$ & $\mathbf{N}$ & $\%$ & $\mathbf{N}$ & $\%$ & \\
\hline \multicolumn{8}{|l|}{ Porte populacional (habitantes) } \\
\hline$<10.000$ & 16 & 8,5 & 20 & 42,6 & 23 & 48,9 & 0,001 \\
\hline 10.000 a 50.000 & 4 & 15,0 & 56 & 56,0 & 29 & 29,0 & \\
\hline$>50.000$ & 6 & 15,1 & 74 & 69,8 & 16 & 15,1 & \\
\hline Condição de habilitação ${ }^{a}$ & 4 & & & & & & \\
\hline GPABA & 4 & 11,8 & 103 & 55,1 & 62 & 33,1 & 0,001 \\
\hline GPSM & 1 & 19,7 & 47 & 71,2 & 6 & 9,1 & \\
\hline
\end{tabular}

${ }^{a}$ condição de gestão dos municípios segundo a Norma Operacional da Assistência a Saúde.

Tabela 3. Distribuição das médias de pontuação percentuais para as avaliações de estrutura e processo do grau de qualidade das salas de vacina em municípios da macrorregião Oeste, Minas Gerais/Brasil, 2011.

\begin{tabular}{|c|c|c|c|c|c|c|}
\hline \multirow{3}{*}{ Dimensões da avaliação } & \multicolumn{6}{|c|}{ Grau de qualidade } \\
\hline & \multicolumn{2}{|c|}{ Adequado } & \multicolumn{2}{|c|}{ Não adequado } & \multicolumn{2}{|c|}{ Crítico } \\
\hline & $\mathbf{N}$ & $\%$ & $\mathbf{N}$ & $\%$ & $\mathbf{N}$ & $\%$ \\
\hline Estrutura & 90 & 35,6 & 133 & 52,6 & 30 & 11,8 \\
\hline Processo geral & 74 & 29,2 & 93 & 36,8 & 86 & 34,0 \\
\hline \multicolumn{7}{|l|}{ Processo por componente } \\
\hline Organização refrigerador & 131 & 51,8 & 25 & 9,9 & 97 & 38,3 \\
\hline Monitoramento temperatura & 179 & 70,8 & 29 & 11,5 & 45 & 17,7 \\
\hline Condutas frente à alteração de temperatura & 135 & 53,3 & 28 & 11,1 & 90 & 35,6 \\
\hline
\end{tabular}

processo. Destaca-se na avaliação de processo o grande número de salas com organização do refrigerador considerado crítico.

Verificou-se que 117 (46,3\%) dos entrevistados realizaram algum tipo de capacitação em sala de vacina. Em relação aos enfermeiros, 60
(43,8\%) realizaram capacitação. Em contrapartida, dos técnicos/auxiliares de enfermagem 57 $(54,8 \%)$ relataram que participaram de capacitações em algum momento.

Também o critério porta vazia que identifica a ausência de material no painel interno do refrige- 
rador foi considerado crítico. Verificou-se a presença de frascos de insulina em $83(32,8 \%)$ refrigeradores, sendo este o objeto mais encontrado.

Os critérios dos três componentes de processo estão apresentados na Tabela 4. Observa-se que o indicador que preenche o formulário de avaliação de imunobiológicos foi o pior critério avaliado no componente condutas frente à alteração de temperatura. Vários profissionais relataram desconhecer o formulário de preenchimento de imunobiológicos sob suspeita, o que pode gerar uma subnotificação de alterações de temperatura, comprometendo a qualidade da rede de frio. Quando investigado sobre os procedimentos relacionados às condutas frente à alteração de temperatura, os profissionais relataram 196 (77,5\%) salas de vacina que comunicam à ins- tância superior, porém em 109 (43,1\%) não há o preenchimento do formulário de imunobiológico sob suspeita e em 99 (39,1\%) não há a manutenção das vacinas em temperatura de $+2^{\circ} \mathrm{C}$ a $+8^{\circ} \mathrm{C}$ até pronunciamento da instância superior.

Outro problema identificado está relacionado ao componente organização do refrigerador. Quanto à disposição das vacinas no interior do refrigerador, observou-se que em 97 (38,3\%) havia a presença de vacinas que não podiam sofrer congelamento dispostas na primeira prateleira. Também foi identificado que em 220 (87,0\%) salas de vacina não existe um programa de manutenção preventiva e/ou corretiva para o refrigerador.

As ações de monitoramento da temperatura apresentaram melhor qualidade em todas as

Tabela 4. Distribuição da média de pontos na avaliação da dimensão estrutura e processo da conservação de vacina, em municípios da macrorregião Oeste do estado de Minas Gerais, 2011.

\begin{tabular}{|c|c|c|}
\hline & $\begin{array}{l}\text { Pontuação } \\
\text { máxima }\end{array}$ & $\begin{array}{l}\text { Média } \\
\text { alcançada }\end{array}$ \\
\hline \multicolumn{3}{|l|}{ Dimensões e componentes } \\
\hline \multicolumn{3}{|l|}{ Estrutura } \\
\hline Existência de refrigerador exclusivo & 1 & 0,8 \\
\hline Existência de termômetro de máxima e mínima & 3 & 2,9 \\
\hline Congelador com gelo reciclável & 1 & 0,9 \\
\hline Parte inferior com garrafas com água & 1 & 0,9 \\
\hline Porta vazia & 1 & 0,3 \\
\hline Profissional capacitado em sala de vacina e/ou rede de frio & 3 & 1,5 \\
\hline \multicolumn{3}{|l|}{ Processo } \\
\hline \multicolumn{3}{|l|}{ Monitoramento da temperatura } \\
\hline $\begin{array}{l}\text { Faz a leitura e registro corretos das temperaturas no início e no final da } \\
\text { jornada de trabalho }\end{array}$ & 3 & 2,5 \\
\hline $\begin{array}{l}\text { Realiza ambientação das bobinas de gelo reciclável na organização da caixa } \\
\text { térmica }\end{array}$ & 1 & 0,9 \\
\hline $\begin{array}{l}\text { Faz o monitoramento da temperatura da caixa térmica ou do equipamento } \\
\text { de uso diário }\end{array}$ & 1 & 0,9 \\
\hline \multicolumn{3}{|l|}{ Organização do refrigerador } \\
\hline $\begin{array}{l}\text { Na } 1^{\text {a }} \text { prateleira são armazenadas em bandejas somente as vacinas que } \\
\text { podem ser submetidas à temperatura negativa. }\end{array}$ & 5 & 3,8 \\
\hline $\begin{array}{l}\mathrm{Na} 2^{\text {a }} \text { prateleira são armazenadas em bandejas somente as vacinas que não } \\
\text { podem ser submetidas à temperatura negativa }\end{array}$ & 2 & 1,7 \\
\hline $\begin{array}{l}\text { O degelo e a limpeza do refrigerador são realizados a cada } 15 \text { dias ou } \\
\text { quando a camada de gelo atinge } 0,5 \mathrm{~cm} .\end{array}$ & 2 & 1,6 \\
\hline $\begin{array}{l}\text { Existe um programa de manutenção preventiva e/ou corretiva para o } \\
\text { refrigerador da sala de vacina }\end{array}$ & 1 & 0,1 \\
\hline \multicolumn{3}{|l|}{ Condutas frente à alteração de temperatura } \\
\hline $\begin{array}{l}\text { Comunica imediatamente a instância hierarquicamente superior em casos } \\
\text { de alterações de temperatura. }\end{array}$ & 5 & 3,9 \\
\hline $\begin{array}{l}\text { Preenche o formulário de avaliação de imunobiológicos sob suspeita e } \\
\text { envia a instância hierarquicamente superior. }\end{array}$ & 3 & 1,7 \\
\hline $\begin{array}{l}\text { As vacinas sob suspeita são mantidas em temperatura de }+2^{\circ} \mathrm{C} \text { a }+8^{\circ} \mathrm{C} \text {, até } \\
\mathrm{o} \text { pronunciamento da instância superior. }\end{array}$ & 2 & 1,2 \\
\hline
\end{tabular}


microrregiões, embora observando-se diferenciações nos percentuais do grau de qualidade nas microrregiões de Itaúna e Campo Belo. Apesar desses resultados, identifica-se que nesse componente o indicador faz a leitura e registro corretos das temperaturas no início e no final da jornada de trabalho também pode ser considerado um entrave.

\section{Discussão}

O Programa Nacional de Imunização brasileiro tem alcançado êxito por meio de altas coberturas vacinais ${ }^{14}$. Porém, é preciso destacar que não basta alcançar altas taxas de coberturas, é preciso também garantir que as vacinas aplicadas estejam com suas propriedades imunizantes preservadas. Para isso é necessário uma série de cuidados com a conservação das mesmas. Os resultados deste estudo mostraram que a qualidade de conservação de vacinas não está consonante às normativas de conservação na maioria dos municípios da região Centro-Oeste de Minas Gerais, sendo as salas de vacina classificadas, na sua maioria, em qualidade não adequada ou crítica.

Evidenciou-se uma concentração de salas consideradas críticas em municípios de pequeno porte. Ao buscar conhecer os fatores condicionantes dessa situação, verificou-se que os municípios menores encontram dificuldades na alocação e capacitação do pessoal, uma vez que nem sempre estes apresentam vínculo estatuário, principalmente o profissional enfermeiro, o que tem propiciado alta rotatividade, dificultando a vinculação e o engajamento necessários para o desenvolvimento das atividades em sala de vacina.

Além disso, em alguns municípios de pequeno porte, é visto o enfermeiro assumindo mais de uma unidade de saúde, o que pode comprometer a supervisão das atividades em sala de vacina e consequentemente a qualidade da conservação. O enfermeiro acaba assumindo responsabilidades e funções, principalmente pela falta de outros profissionais, acumulando atribuições que interferem na finalidade do serviço e no desenvolvimento das atividades ${ }^{15}$.

Considera-se que o cotidiano de trabalho do enfermeiro na sala de vacina perpassa a organização tecnológica e social do trabalho, verificando-se um quadro dialético e contraditório em que se vivencia o processo de trabalho de enfermagem/ enfermeira, e, consequentemente, o exercício da supervisão face à diversidade e à dinamicidade de suas ações ${ }^{16}$. Enfermeiros desempenham papel essencial na garantia de melhor conservação de vacinas ${ }^{17}$.

Considerando os resultados referentes à estrutura, constata-se uma falta de capacitação para os profissionais que exercem atividades em sala de vacina. O PNI ampliou sistematicamente o número de vacinas disponibilizadas, nos últimos anos, e esta introdução de novas vacinas, traz no bojo uma série de necessidades de conhecimento como: vias de administração, conservação, possíveis reações adversas, manuseio, dentre outras. Estas mudanças requerem do profissional uma constante atualização em sala de vacina, além de visão crítica e reflexiva do seu trabalho. Neste sentido, a educação permanente em saúde foi introduzida com a finalidade de aproximar a educação do ambiente de trabalho, incorporando o ensino e o aprendizado à vida cotidiana das organizações e às práticas sociais e laborais, no contexto real em que ocorrem ${ }^{18}$.

Ainda referente à estrutura, também se identificou em algumas salas de vacina o armazenamento de outros materiais no refrigerador, o que expõe as vacinas a riscos, tanto de contaminação devido ao aumento da manipulação do equipamento, quanto de maior exposição à variação da temperatura, ao abrir o refrigerador várias vezes levando à oscilação da temperatura.

Além disso, os refrigeradores domésticos, apesar da facilidade de aquisição e baixo custo, não foram projetados para manutenção da temperatura na escala requerida, pois não apresentam homogeneidade na distribuição térmica, o termostato reage à temperatura do evaporador $\mathrm{e}$ não ao ar geral no compartimento interno. Assim, a temperatura média pode variar com a do ambiente externo, e estes fatores podem aumentar o risco de perdas e comprometer a confiabilidade do produto ofertado ${ }^{19}$.

Os resultados referentes ao processo identificaram que a organização do refrigerador pode ser considerada um ponto crítico nas salas de vacina da região. As vacinas no nível local são conservadas em temperatura entre $+2^{\circ} \mathrm{C} \mathrm{e}+8^{\circ} \mathrm{C}$, e são distribuídas no refrigerador de acordo com a termoestabilidade, ou seja, as vacinas que contêm adjuvantes não podem ser submetidas a congelamento e assim são dispostas na segunda prateleira.

É importante mencionar que achados incongruentes sobre a disposição dos imunobiológicos no interior do refrigerador também foram encontrados em outros estudos brasileiros ${ }^{3,20,21}$.

Em caso de alterações de temperatura, o PNI define algumas normas que devem ser seguidas para evitar perdas desnecessárias de vacinas e 
administração de vacinas inativadas, são elas: comunicar imediatamente à instância hierarquicamente superior; preencher o formulário de avaliação de imunobiológico sob suspeita e manter as vacinas sob suspeita em temperatura de $+2^{\circ} \mathrm{C}$ $\mathrm{a}+8^{\circ} \mathrm{C}$, até o pronunciamento da instância superior $^{22}$. Em nosso estudo estas recomendações foram avaliadas no componente, Condutas frente à alteração de temperatura, o qual obteve os piores percentuais de avaliação.

O cumprimento destas recomendações evita o desperdício desnecessário e falhas vacinais por inativação de produtos, pois não existe um método simples e barato para avaliar se uma vacina exposta a alterações de temperatura ainda retém a potência mínima requerida. A potência de uma vacina só pode ser determinada por ensaios laboratoriais de alto custo. Sendo assim, somente um grande número de doses justifica o envio de uma vacina para reteste ${ }^{23}$. A prevenção de alterações de temperatura das vacinas faz-se necessário tanto para diminuir gastos de dinheiro como para assegurar que estas estejam plenamente potentes e eficazes ${ }^{24}$.

No componente Monitoramento da temperatura também foi identificado como limitação a leitura e o registro corretos das temperaturas no início e no final da jornada de trabalho. Devemos levar em consideração que o mapa de controle diário da temperatura oferece informações sobre a variação da temperatura do refrigerador, além de ser considerado um documento que valida a qualidade da refrigeração dos imunobiológicos.

Identifica-se que o problemas na conservação de vacinas não é exclusividade do Brasil. Estudos realizados em países desenvolvidos, para avaliar a cadeia de frio, encontraram 23,3\% de unidades públicas de saúde de Madrid ${ }^{25}$ que não realizavam controle diário da temperatura, e em Valência ${ }^{26}, 75 \%$ dos centros de saúde não preenchiam diariamente o gráfico de controle de temperatura. Além disso, em 33,8\% das geladeiras havia presença de alimentos e em 39,7\% dos refrigeradores as vacinas não estavam dispostas corretamente ${ }^{26}$.

No mesmo raciocínio, uma investigação realizada no Nordeste do Brasil para caracterizar a experiência e o conhecimento da equipe de enfermagem sobre imunização identificou que em $75 \%$ das unidades de saúde, o mapa de registro diário da temperatura do refrigerador continha valores mínimos de $0,9^{\circ} \mathrm{C}$ e máximo de $18,9^{\circ} \mathrm{C}$, que são contestados pelo $\mathrm{PNI}^{27}$.

Pode-se reconhecer limitações no presente estudo. Inicialmente, existe uma possibilidade de o indicador "profissional capacitado" ter influenciado negativamente a classificação geral das salas de vacina. A sala de vacina, mesmo apresentando todos os indicadores selecionados na estrutura, se não existir o profissional capacitado ela perde $30 \%$ no componente estrutura, o que a classificaria como não adequada ou crítica. Outra consideração é o delineamento utilizado. Por ser uma pesquisa avaliativa de serviços, os resultados devem ser discutidos considerando-se a realidade local. Outro aspecto importante foi a escassez de trabalhos realizados e divulgados sobre a qualidade da conservação de vacina, principalmente no Brasil, o que de certa forma dificultou uma discussão mais aprofundada sobre os resultados. Apesar das limitações aqui expostas, os aspectos levantados neste estudo permitem uma aproximação e uma ideia geral da situação da qualidade da conservação de vacina na Região Centro-Oeste de Minas Gerais, ao mesmo tempo em que destacam os desafios a serem enfrentados.

\section{Considerações finais}

A qualidade da conservação de vacinas não está adequada na maioria dos municípios da macrorregião Oeste de saúde de Minas Gerais. Esta avaliação aponta para a deficiência de fatores relacionados à estrutura e ao processo que podem influenciar na efetividade do PNI na região, podendo citar: a insuficiência de recursos humanos capacitados, falhas na organização do refrigerador, no monitoramento da temperatura e no cumprimento das orientações em caso de alteração de temperatura do refrigerador da sala de vacina.

Apesar dos avanços do PNI e dos impactos positivos reconhecidos mundialmente, ainda observamos falhas na estrutura das salas de vacina como o uso do refrigerador do tipo doméstico como equipamento predominante, o que exige maiores cuidados e atenção da equipe de enfermagem e expõe os imunobiológicos ao risco de alterações de temperatura. Associado a isso, uma falta de manutenção desses equipamentos pode acarretar prejuízos ao PNI por perda desnecessária de imunobiológicos.

O PNI é referência internacional em imunização, devido aos seus avanços na prevenção, controle e eliminação de doenças imunopreveníveis. Entretanto, é no nível local que a operacionalização das ações acontece e, portanto, a manutenção da qualidade do imunobiológico administrado na população. Nesse sentido, é im- 
prescindível uma adequada estrutura nas UAPS que favoreça o desenvolvimento do processo de conservação de vacinas e, consequentemente, a manutenção de resultados efetivos no PNI.

\section{Colaboradores}

VC Oliveira participou da concepção, planejamento, coleta, análise dos dados, elaboração do manuscrito e aprovação final. TL Gontijo contribuiu com a interpretação dos resultados, elaboração do artigo e aprovação final. RA Arcêncio na revisão crítica do conteúdo, revisou o texto final do artigo. MDPS Gallardo colaborou no desenho metodológico, interpretação dos dados e elaboração da versão final do artigo. IC Pinto participou de todas as etapas como orientadora do projeto e versão final do artigo.

\section{Agradecimentos}

À Coordenação de Aperfeiçoamento de Pessoal de Nível Superior/CAPES no fornecimento de bolsa de doutorado sanduíche na Universidade Autônoma de Madrid/UAM. 


\section{Referências}

1. Guimarães TMR, Alves JGB, Tavares MMF. Impacto das ações de imunização pelo Programa Saúde da Família na mortalidade infantil por doenças evitáveis em Olinda, Pernambuco, Brasil. Cad Saude Publica 2009; 25(4):868-876.

2. Homma A, Martins RM, Leal MLF, Freire MS, Couto AR. Atualização em vacinas, imunizações e inovação tecnológica. Cien Saude Colet 2011; 16(2):445-458.

3. Melo GKM, Oliveira JV, Andrade MS. Aspectos relacionados à conservação de vacinas nas unidades básicas de saúde da cidade do Recife - Pernambuco. Epidemiol Serv. Saúde 2010; 19(1):25-32.

4. Santos DM, Dubeux LS, Frias PG, Vanderlei LCM, Vidal SA. Avaliação normativa da ação programática Imunização nas equipes de saúde da família do $\mathrm{Mu}$ nicípio de Olinda, Estado de Pernambuco, Brasil, em 2003. Epidemiol. serv. saúde 2006; 15(3):29-35.

5. Oliveira VC, Guimarães EAA, Guimarães IAG, Januário LH, Pinto IC. Prática da enfermagem na conservação de vacinas. Acta paul. enferm 2009; 22(6):814-818.

6. Oliveira VC, Guimarães EAA, Silva SS, Pinto IC. Conservação de vacinas em unidades básicas de saúde: análise diagnóstica em municípios mineiros. Rev Rene 2012; 13(3):531-541.

7. Brasil. Ministério da Saúde (MS). Secretaria de Vigilância em Saúde. Departamento de Vigilância Epidemiológica. Manual de manutenção de equipamentos da rede de frio. Brasília: MS; 2007.

8. Aranda CMSS, Moraes JC. Rede de frio para a conservação de vacinas em unidades públicas do município de São Paulo: conhecimento e prática. Rev. bras. Epidemiol 2006; 9(2):172-185.

9. Mccolloster P, Vallbona C. Graphic-output temperature data loggers for monitoring vaccine refrigeration: implications for pertussis. Am J Public Health 2011; 101(1):46-48.

10. Tanaka OY, Tamaki EM. O papel da avaliação para a tomada de decisão na gestão de serviços de saúde. Cien Saude Colet 2012; 17(4):821-828.

11. Instituto Brasileiro de Geografia e Estatística (IBGE). Censo demográfico de 2012. [acessado 2012 nov 17]. http://www.ibge.gov.br

12. Champagne F, Brouselle A, Hartz Z, Contandriopoulos AP. Modelizar as intervenções. In: Brouselle A, Champagne F, Contandriopoulos AP, Hartz Z. Avaliação: conceitos e métodos. Rio de Janeiro: Fiocruz; 2011. p. 61-76.

13. Brasil. Ministério da Saúde (MS). Sistema de Informação do Programa Nacional de Imunização (SI-PNI). Programa de Avaliação do Instrumento de Supervisão Salas de Vacina (PAISSV). Secretaria Executiva. Manual do usuário. Rio de Janeiro: MS; 2003.

14. Figueiredo GLA, Pina JC, Tonete VLP, Lima RAG, Mello DF. Experiências de famílias na imunização de crianças brasileiras menores de dois anos. Rev. Latino -Am. Enfermagem [internet]. maio-jun 2011 [acessado 2013 abr 28];19(3):[08 telas]. Disponível em: http:// www.scielo.br/pdf/rlae/v19n3/pt_20.pdf
15. Ferraz LNS, Santos AS. O programa de saúde da família e o enfermeiro: atribuições previstas e realidade vivencial. Saúde Coletiva 2007; 4(15):89-93.

16. Servo MLS. Pensamento estratégico: uma possibilidade para a sistematização da supervisão em enfermagem. Rev Gaúcha Enferm 2001; 22(2):39-59.

17. Carr C, Byles J, Durrheim D. Practice nurses best protect the vaccine cold chain in general practice. Aust $J$ Adv Nurs 2010; 27(2):35-39.

18. Brasil. Ministério da Saúde (MS). Secretaria de Gestão do Trabalho e da Educação na Saúde. Departamento de Gestão da Educação em Saúde. Política Nacional de Educação Permanente em Saúde. Brasília: MS; 2009.

19. São Paulo. Secretaria Municipal da Saúde (SMS). Coordenação da Vigilância em Saúde. Centro de Prevenção e Controle de Doenças. Subgerência de Imunização. Norma técnica do programa de imunização. São Paulo: SMS; 2008.

20. Araújo ACM, Silva MRF, Frias PG. Avaliação da Rede de Frio do Programa Municipal de Imunização do Distrito Sanitário IV do Município do Recife. Rev APS 2009; 12(3):238-242.

21. Queiroz SA, Moura ERF, Nogueira PSF, Oliveira NC, Pereira MMQ. Atuação da equipe de enfermagem na sala de vacinação e suas condições de funcionamento. Rev Rene 2009; 10(4):126-135.

22. Brasil. Ministério da Saúde (MS). Secretaria de Vigilância em Saúde. Departamento de Vigilância Epidemiológica. Manual de rede de frio. Brasília: MS; 2007.

23. World Health Organization (WHO). Temperature sensitivity of vaccines. Geneva: WHO; 1988.

24. Matthias DM, Robertson J, Garrison MM, Newland S, Nelson C. Freezing temperatures in the vaccine cold chain: a systematic literature review. Vaccine 2007; 25(20):3980-3986.

25. Arbiza PA, Vicente RA, Rábago MLG, Rojas VD, Molina, PO, Pardo JRJ. Cadena del frío para la conservación de las vacunas en los centros de atención primaria de un area de Madrid: mantenimiento y nível de conocimientos. Rev Esp Salud Pública 2002; 76(4):333-346.

26. Hueso BC, Sánchez OR, Pérez IC, Peiró S. The vaccine cold chain in a Valencian health departamento (Spain). Gac Sanit 2009; 23(2):139-143.

27. Luna GLM, Vieira LJES, Souza PF, Lira SVG, Moreira DP, Pereira AS. Aspectos relacionados à administração e conservação de vacinas em centros de saúde no Nordeste do Brasil. Cien Saude Colet 2011; 16(2):513-521.

Artigo apresentado em 20/05/2013

Aprovado em 16/07/2013

Versão final apresentada em 20/07/2013 